REGARDS

SUR L'ECONOMIE ALLEMAND

BULLETIN ECONOMIQUE DU CIRAC
Regards sur l'économie allemande

Bulletin économique du CIRAC

113 | 2014

Varia

\title{
Sur les sentiers de la croissance
}

Isabelle Bourgeois

\section{OpenEdition}

Journals

Édition électronique

URL : http://journals.openedition.org/rea/4680

DOI : $10.4000 /$ rea. 4680

ISSN : 1965-0787

Éditeur

CIRAC

Édition imprimée

Date de publication : 15 juillet 2014

Pagination : $3-4$

ISSN : 1156-8992

\section{Référence électronique}

Isabelle Bourgeois, «Sur les sentiers de la croissance», Regards sur l'économie allemande [En ligne], 113 | juillet 2014, mis en ligne le 17 juillet 2016, consulté le 15 septembre 2020. URL : http:// journals.openedition.org/rea/4680 


\section{Sur les sentiers de la croissance}

L'économie allemande semble avoir trouvé sa vitesse de croisière. Après avoir " démarré l'année en trombe », comme le formule la banque KfW (27-05), elle confirme sa robustesse. Au total, la croissance 2014 devrait atteindre 1,8\% selon le gouvernement fédéral, $2 \%$ selon la banque KfW ou "près de $2 \%$ » selon la Bundesbank qui résume dans son rapport de juin : "les conditions sont réunies pour une croissance relativement soutenue », et ce d'ici 2016.

Au $1^{\mathrm{er}}$ trimestre 2014 , le PIB a connu une croissance de 0,8\% par rapport au précédent $(+0,4 \%)$, mais de $2,5 \%$ en un an (Destatis). Une telle croissance n'a pas été recensée depuis trois ans. L'hiver très doux n'y est pas étranger, la Bundesbank estimant que 0,3 point de pourcentage est à mettre à son actif. De ce fait, la reprise au début du printemps devrait être légèrement plus faible que d'habitude durant cette période. Mais la dynamique de croissance reste foncièrement solide.

Au fil des mois, le moteur de la production manufacturière qui tirait la croissance à la fin 2013 a été relayé au début de l'année par le BTP et le secteur des services, tandis que la consommation intérieure a pris le relais des exportations. Au premier trimestre 2014 , les importations ont progressé nettement plus fort $(+2,2 \%)$ que les importations $(+0,2 \%)$, et la contribution de la balance commerciale au PIB a été négative $(-0,9$ point de pourcentage ; Destatis). La valeur des échanges n'en reste pas moins importante : elle atteignait un peu plus de 355 milliards $€$ en données CVS pour les exportations (contre près de 340 milliards $€$ au $1^{\text {er }}$ trimestre 2013) et près de 314 milliards $€$ pour les importations (contre 303 milliards $€$ ). Et, en comparaison annuelle, la part de l'excédent commercial dans le PIB a été de 0,1 point au début 2014. Le solde négatif du premier trimestre n'a rien d'inquiétant, au contraire, puisqu'il est dû à une forte demande intérieure allemande qui tire les importations, "ce qui tire la demande de biens en provenance des pays de la zone Euro et soutient la reprise de leur économie », comme ne manque pas de le souligner la banque KfW.

La consommation intérieure confirme ainsi son rôle de nouveau moteur conjoncturel. Considéré globalement, cet état de fait consolide aussi l'économie allemande, puisqu'il la " protège jusqu'à un certain point contre le ralentissement conjoncturel principalement dans les économies émergentes et contre les risques émanant du contexte mondial ", rappelle également la banque KfW. II permet en tout cas d'amortir quelque peu les risques liés à la crise en Ukraine et en Irak qui, jusqu'ici, n'affectaient pas le moral des milieux d'affaires, y compris dans les secteurs exportateurs. Les entreprises se montrent optimistes, leurs carnets de commande sont en effet bien remplis.

La consommation des ménages a été en hausse de $0,7 \%$ au $1^{\text {er }}$ trimestre, les dépenses de consommation de l'Etat de 0,4\%. Le BTP a profité de l'hiver clément et de l'engouement des ménages pour la construction immobilière $(+3,8 \%$; KWW). Quant aux investissements, ils étaient plus soutenus au niveau des pouvoirs publics que des entreprises (respectivement $+11,6 \%$ et $+1,6 \%$; KfW). Ils ont plus progressé dans le domaine de la construction que de l'équipement (respectivement $+3,6 \%$ et 3,3\%; Destatis) ; en comparaison annuelle, la hausse est particulièrement nette, avec respectivement $+10,2 \%$ et $+6,0 \%$.
$+0,8 \%$

au ${ }^{\text {er }}$ trimestre 2014

Le déficit commercial soutient la reprise dans la zone Euro

Effet stabilisateur de la consommation intérieure

\footnotetext{
Les données du $1^{\text {er }}$ trimestre 2014 publiées par Destatis sont établies conformément au système européen des comptes nationaux SEC 95. A partir de l'été, il sera remplacé dans l'ensemble de l'UE par un nouveau système, le SEC 2010. C'est sur cette nouvelle base que Destatis publiera le 14-08 ses statistiques relatives au $2^{\mathrm{e}}$ trimestre et révisera l'ensemble des comptes nationaux allemands depuis 1991.
}

$\mathrm{Si}$, parmi tous les secteurs, c'est le BTP qui a connu la plus forte croissance $(+7,0 \%)$ L'industrie embauche au $1^{\text {er }}$ trimestre, suivi de l'agriculture, de la sylviculture et de la pêche $(+6,7 \%)$, l'industrie vient en troisième position $(+4,8 \%)$. Elle génère toujours la plus grande part des richesses outre-Rhin (près de 133 milliards $€$ ), les deux autres secteurs ne 'pesant' que respectivement 28 milliards $€$ et moins de 5 milliards $€$. L'industrie continue également à embaucher : en avril, le nombre de salariés a augmenté de 0,9\% pour atteindre un total de 5,3 millions (entreprises de plus de 50 salariés). Les branches qui ont le plus recruté en avril sont la production d'installations électriques, le secteur automobile et la plasturgie $(+2,2 \%$ chacun $)$. 
Bonne tenue de l'emploi

Heures travaillées niveau historique

Salaires réels en hausse

Bund et Länder de l'Est : respect de la 'règle d'or' budgétaire
En mai, le nombre des actifs occupés avait augmenté de 391000 personnes en un an, passant à désormais 42,1 millions (Destatis), principalement sous l'effet des migrations au sein de l'UE qui compensent légèrement le recul de la main-d'œuvre lié au vieillissement démographique, que va accentuer la réforme des retraites, entrée en vigueur le $1^{\text {er }}$ juillet 2014, qui facilite les départs à la retraite dès 63 ans pour ceux qui ont cotisé 45 ans. Quant au taux de chômage, il se situait à 5,1\% en données CVS (critères OIT) ou à 6,5\% en données administratives. Le nombre de chômeurs indemnisés était de 2,833 millions (Agence fédérale pour l'emploi). Le marché du travail reste bien orienté : d'avril à mai, le nombre d'actifs occupant un emploi régulier (soumis à cotisations sociales) a progressé de 129000 , portant le total de ces emplois à 29,64 millions. L'Agence de Nuremberg recense en juin 462000 postes à pourvoir, essentiellement dans la vente, la mécatronique, la santé, l'hôtellerie/gastronomie, les transports et la logistique.

Quant au volume des heures travaillées, il atteint au $1^{\text {er }}$ trimestre « son plus haut niveau depuis 22 ans ", constate l'Institut IAB près de l'Agence de Nuremberg : 15 milliards d'heures (soit $+2,8 \%$ en un trimestre). Le nombre d'actifs occupés a augmenté de $0,8 \%$, et le nombre d'heures travaillées par actif, de $2 \%$. En moyenne, les salariés à temps plein ont travaillé 38 heures par semaine, 15 heures en temps partiel ; chaque salarié a effectué 11,6 heures complémentaires rémunérées.

$\mathrm{Au} 1^{\mathrm{er}}$ trimestre, les salaires réels ont augmenté de 1,3\% par rapport à la même période de 2013 où, selon les calculs de Destatis, le salaire horaire brut (hors primes) se situait en moyenne, temps pleins et temps partiels confondus, à 19,65 $€$. Plus précisément, durant les trois premiers mois de l'année, les salaires conventionnels ont augmenté de 3,3\%, et cette tendance devrait se poursuivre au fil du calendrier 2014 des négociations tarifaires de branche (Bundesbank, rapport de mai). Face à une situation de quasi plein emploi, une inflation modérée et une rémunération faible des placements financiers, et remplis de confiance face à la robustesse de leur économie, les Allemands ont le moral et consomment. Cette tendance devrait se poursuivre, et la Bundesbank s'attend même à ce que jusqu'en 2016, leurs dépenses augmentent globalement plus vite que leur revenu disponible (rapport de juin).

Les Allemands sont également confortés dans leur optimisme serein par la stabilité des finances publiques : la Fédération respecte la 'règle d'or', présentant un budget largement à l'équilibre, ce qui lui permet de lancer son programme d'investissement dans la modernisation et le développement des infrastructures ; en 2014, elle n'a programmé que 6,5 milliards $€$ de nouvel endettement. Quant aux Länder, leurs budgets ne sont pour la plupart réellement en cours d'équilibre qu'à l'est de l'Allemagne, où la conscience des enjeux semble plus prononcée qu'à l'ouest. Pour les nouveaux Länder, la contrainte est particulièrement forte en effet : leur croissance ayant développé une dynamique propre, ils ne pourront bientôt plus bénéficier des fonds structurels européens ni non plus des fonds qu'ils perçoivent au titre de la transition (Aufbau Ost) dont la fin est programmée en 2019 ; et en 2020, la Constitution leur interdit tout recours au nouvel endettement. Ils mènent donc, quelle que soit l'obédience de leurs gouvernements, une politique prévoyante et rigoureuse de consolidation budgétaire.

L'ÉCONOMIE ALLEMANDE SE PORTE BIEN, MAIS les perspectives de croissance sont entachées par un certain nombre de risques extérieurs et, bien plus encore, intérieurs. Aux tensions géopolitiques s'ajoute la menace d'une nouvelle crise de confiance au sein de la zone Euro. En interne, la réforme des retraites pourrait assécher, surtout dans le Mittelstand, le vivier des compétences et de l'aptitude à innover que représentent les salariés les plus âgés. Le salaire minimum légal de 8,50€ adopté par le Bundestag le 3 juillet et qui devrait concerner près de 4 millions d'actifs pourrait reconstituer un stock incompressible de chômeurs de longue durée parmi les moins qualifiés. De même, le travail non déclaré pourrait connaître une nette hausse. Ces évolutions risquent de réduire in fine l'encours fiscal, de remettre en cause la soutenabilité des régimes de protection sociale et d'accroître les dépenses publiques liées à la lutte contre le chômage. Plus particulièrement, elles risquent de pénaliser les économies est-allemandes où se concentrent nombre d'emplois faiblement qualifiés. A quoi s'ajoute une spirale inflationniste des salaires, de nature à bousculer l'équilibre sectoriel sur lequel reposent l'économie allemande et son rôle "d'ancre de stabilité » au sein de l'UE.

I. Bourgeois (04-07-2014) 